\title{
Overview of Chinese Dynasties
}

\begin{tabular}{|c|c|c|c|c|c|}
\hline \multicolumn{3}{|c|}{ Reign } & \multicolumn{3}{|c|}{ Gregorian Calendar } \\
\hline \multicolumn{3}{|c|}{ Xia Dynasty } & \multicolumn{3}{|c|}{$2070-1600 \mathrm{BC}$} \\
\hline \multicolumn{3}{|c|}{ Shang Dynasty } & \multicolumn{3}{|c|}{ 1600-1046 BC } \\
\hline \multirow{3}{*}{$\begin{array}{l}\text { Zhou } \\
\text { Dynasty }\end{array}$} & \multicolumn{2}{|c|}{ West Zhou } & \multirow{3}{*}{$\begin{array}{l}1046-221 \\
\text { BC }\end{array}$} & \multicolumn{2}{|c|}{ 1046-771 BC } \\
\hline & \multirow[t]{2}{*}{$\begin{array}{l}\text { East } \\
\text { Zhou }\end{array}$} & $\begin{array}{l}\text { Spring } \\
\text { and } \\
\text { Autumn } \\
\text { Period }\end{array}$ & & \multirow{2}{*}{$\begin{array}{l}770-221 \\
\text { BC }\end{array}$} & $\begin{array}{c}770-476 \\
\text { BC }\end{array}$ \\
\hline & & $\begin{array}{c}\text { Warring } \\
\text { States } \\
\text { Period }\end{array}$ & & & $\begin{array}{c}475-221 \\
\text { BC }\end{array}$ \\
\hline \multicolumn{3}{|c|}{ Qin Dynasty } & \multicolumn{3}{|c|}{$221-207 \mathrm{BC}$} \\
\hline \multicolumn{3}{|c|}{ Han Dynasty } & \multicolumn{3}{|c|}{$206 \mathrm{BC}-\mathrm{AD} 220$} \\
\hline \multicolumn{3}{|c|}{ Three Kingdoms } & \multicolumn{3}{|c|}{ AD 220-265 } \\
\hline \multicolumn{3}{|c|}{ Jin Dynasty } & \multicolumn{3}{|c|}{ AD $265-420$} \\
\hline \multicolumn{3}{|c|}{ Southern and Northern Dynasties } & \multicolumn{3}{|c|}{ AD 420-589 } \\
\hline \multicolumn{3}{|c|}{ Sui Dynasty } & \multicolumn{3}{|c|}{ AD 581-618 } \\
\hline \multicolumn{3}{|c|}{ Tang Dynasty } & \multicolumn{3}{|c|}{ AD 618-907 } \\
\hline \multicolumn{3}{|c|}{$\begin{array}{c}\text { Five Dynasties and Ten } \\
\text { Kingdoms }\end{array}$} & \multicolumn{3}{|c|}{ AD 907-960 } \\
\hline \multicolumn{3}{|c|}{ Song Dynasty } & \multicolumn{3}{|c|}{ AD 960-1279 } \\
\hline \multicolumn{3}{|c|}{ Yuan Dynasty } & \multicolumn{3}{|c|}{ AD 1271-1368 } \\
\hline \multicolumn{3}{|c|}{ Ming Dynasty } & \multicolumn{3}{|c|}{ AD 1368-1644 } \\
\hline \multicolumn{3}{|c|}{ Qing Dynasty } & \multicolumn{3}{|c|}{ AD 1644-1911 } \\
\hline
\end{tabular}

Source: Data retrieved from 中国政府门户网站 [www.gov.cn], 《中国历史概况》 [Overview of Chinese History], 24 May $2005<\mathrm{http} / / / \mathrm{www} . g o v . c n / t e s t / 2005-05 / 24 /$ content_436.htm>. 